\title{
EDITORIAL
}

\section{SEXUALITY, AGING, AND DEMENTIA}

\author{
S. RECTOR ${ }^{1}$, S. STIRITZ1 , J.E. MORLEY ${ }^{2}$ \\ 1. George Warren Brown School of Social Work, Washington University, St. Louis, Missouri, USA; 2. Division of Geriatric Medicine, Saint Louis University School of Medicine, St. \\ Louis, Missouri USA. Corresponding author: John E. Morley, MB, BCh, Division of Geriatric Medicine, Saint Louis University School of Medicine, 1402 S. Grand Blvd., M238, St. \\ Louis, MO 63104,Email: john.morley@health.slu.edu
}

\section{"Sex doesn't disappear \\ It just changes forms" \\ $\sim$ Erica Jong}

Sexual health is an important facet of life and overall wellbeing for individuals of all ages and abilities. A 2007 data analysis of the National Social Life, Health, and Aging Project found that the majority of older adults surveyed were engaged in intimate relationships and viewed sexuality as an important part of their lives (1). About three quarters of couples 57 to 64 years of age have sexual intercourse with numbers declining to about a quarter aged 75 to 85 (1) There are many healthrelated issues that can impact sexual activity among older adults as they age. A broader definition of sex is needed to better understand the sexual lives of older adults. The major problems reported are loss of lubrication and poor climax in women and poor libido and erectile dysfunction in men. Lack of a partner who is capable of or enthused about sex remains the major problem for many older adults (2). Older persons who participate in sexual activity are more likely those who are physically active (3). The concept of "couple pulse" highlights the need for health professionals to recognize the need to help both members of the social dyad and not to focus on only one person (4). Sexuality is not limited to intercourse related activities but also includes hand-holding, petting, kissing, hugging, and flirting (5). Sexual activity and intimacy among older adults have also been associated with greater enjoyment of life and an increase in overall well-being (6). Although there is ample evidence to suggest that sexuality is an important facet of health across the life course, it is often ignored when caring for older adults. This is especially true when older persons develop changes in cognitive functioning or transfer to long-term care setting. Transitioning into long-term care is often accompanied by a loss of autonomy, and residents are subject to the values and biases of staff and other healthcare providers. This loss of independence can become exacerbated for individuals with dementia.

While residential care facilities (care homes) claim to focus on providing the best possible quality of life for their residents with dementia, many do not consider the role of sexual health within the daily lives of residents (7).

While sexual health plays a vital role in the well-being of older adults, creating a safe space for those with dementia to explore their sexuality comes with complex challenges. Interventions created to protect the sexual rights of those with dementia living in long-term care settings should utilize a sexual health through the life course perspective. Sexual development is a lifelong process, and the presence of a degenerative disease associated with dementia does not equate to the end of ones sexuality.

Healthcare professionals often neglect to acknowledge the sexual lives of their older adult patients. An increase in patient age is associated with a decrease in documenting a patient's sexual history (8). Physician barriers to discussing sexual health include pervasive stereotypes of sexuality and aging rather than the health care needs and experiences of their patients (9). This neglect of sexual health considerations can be exacerbated for those with dementia. It is important that health care professionals give their patients permission to discuss their sexuality and, with the consent of the patient, obtain as detailed of an individual's sexual history as possible. Without consideration for an individual's sexual health, health care providers will not have a holistic view of the patient's needs and may be neglecting issues that are negatively impacting their overall health and well-being.

It is only in the last 30 years that physicians have begun to aggressively treat changes in sexual functioning. This has occurred because of the availability of different formulations of testosterone to treat low libido in those with penises and to a lesser extend in those with vulvas (10-12). The recognition that phosphodiesterase inhibitors could improve erectile dysfunction was made in 1998 (13) and sildenafil and other agents became available ten years later $(14,15)$. Other treatments for erectile dysfunction such as penile prostheses, penile injections and vacuum tumescence devices also became available (16-18). When physicians neglect to take sexual histories and discuss sexual health issues with their patients, they are also less likely to be treating changes in sexual functioning. Older adult patients and those with dementia may be interested in exploring the medical interventions discussed here.

\section{Sexual Activity and Dementia}

Few studies research the prevalence of sexual activity and intimacy among those with dementia. In persons with dementia living at home $48 \%$ of males and $18 \%$ of females with dementia were sexually active (19). In a study by Holmes 


\section{THE JOURNAL OF NUTRITION, HEALTH \& AGING}

et al (20), a survey on the prevalence of sexual activity among nursing home residents was distributed to nursing home staff. Respondents reported that $16 \%$ of males and $10 \%$ of females had engaged in some form of sexual activity while living at the facility. Sexual activity as defined in this study included masturbation, genital and non-genital touching, and sexual intercourse (20). A more recent study of communitydwelling heterosexual spousal couples, of which one partner was diagnosed with Alzheimer's disease, found that $70 \%$ of respondents reported practicing physically intimate activities. These activities included kissing, hugging, and intercourse (21). It is important to note that the findings also reported that sexual intercourse was found to be difficult for the majority of spouses experiencing dementia. Researchers hypothesized that these challenges could be due to difficulties with following the sequencing of events or losing focus and arousal. Both studies provide evidence that among those with dementia, the desire for sexual activity and intimacy is still present. These studies also provide evidence that the definitions of sexual activity should be expanded to include more facets of sexuality beyond sexual intercourse or genital arousal.

Numerous studies regarding sexuality and dementia define most sexual activity as inappropriate sexual behaviors (22) though in a modern context this would not seem appropriate. While this may be true with seemingly out of control sexual behavior that can manifest in some cases of variant frontotemporal dementia, there is discord among researchers on what should be considered appropriate and inappropriate sexual behaviors in association with dementia. Some suggest that sexual behaviors are a result of disinhibition caused by dementia symptoms and can include sexual touching, disrobing, masturbation, sexual advances, and implied manifestations (such as reading pornographic materials) $(23,24)$. These definitions lack context and seemingly relate most sexual behaviors among those with dementia as burdensome and stressful to families and caregivers. Wilkins (25) provides more context in defining inappropriate sexual behaviors. Wilkins states:

"Inappropriate sexual behaviors can be defined as behaviors that are unsafe, disruptive, and impair the care of the resident; in this context, these behaviors include sexually explicit language, sexual acts (touching, grabbing, exposing, masturbating in public), and implied sexual acts (reading sexually explicit material, requests for unnecessary care)."

Limited definitions for appropriate sexual behaviors exist, and defining this term can be difficult given the subjective nature of what constitutes appropriate behavior for individuals in specific settings. Wilkins (25) acknowledges this challenge, but states that "one can assume that appropriate sexual contact implies consensual sexual contact." Defining consent in the presence of dementia is a complex issue which will be discussed further in this editorial.

\section{Barriers to Sexual Expression}

The transition to long-term residential care is often marked with a loss of independence that can negatively impact the sexual health of those living in these settings. There are a number of barriers that diminish a resident's sexual expression, including lack of privacy, difficulty finding a partner, chronic illness, decreased self-esteem, values and attitudes of the facility staff and health care providers, and a limited understanding of sexuality (26). While many long-term care facilities are turning to a person-centered approach, many are neglecting to acknowledge the importance of sexuality in the lives of their residents (25).

A long-term care facility's values related to the sexual rights of residents are reflected in their policies and guidelines for practice. In a 2016 national study of 366 skilled nursing facilities, researchers identified facility policies related to sexual activity and sexual relationships through a survey that was distributed to directors of nursing (26). The study found that although the vast majority $(71 \%)$ of respondents identified that their facility had experienced issues related to residents' sexual activity, more than half $(63 \%)$ of the facilities did not have policies in place to address resident sexual activity. Of the facilities that did have written policies regarding the sexual activity or sexual rights of the residents, $52 \%$ applied the policy to all residents, $22 \%$ specifically mentioned residents with cognitive impairment, and $22 \%$ applied the policy in sexual activity as requested. These results show that there is a need for specific policies that discuss the sexual rights of residents in long-term care settings.

Residents living in facility settings are subjected to the beliefs, values, and biases held by staff. In many cases, caregivers act as gatekeepers in how much autonomy residents may have in most areas of their lives (27). In a study of 100 nursing home staff from three facilities in Alabama, knowledge and perceptions of sexuality among older adults in residential care settings were analyzed (7). The majority of participant's reported neutral attitudes towards sexual activity among residents but did express concern for sexual activity between residents with dementia. These neutral attitudes only reflected heteronormative sexual expression between residents without dementia. Participants' attitudes were more negative when the sexual activity occurred between residents of the same sex. In some cases, this is magnified by the attitudes of the children of these residents. It must be recognized that same sex interest in an older adult with dementia may represent a lifetime yearning and identity hidden from family. LGBTQ older adults often face added layers of oppression in health care settings. Stigma and discrimination make finding a safe and affirmative residential care environment especially challenging. Besides the fear of discrimination, LGBTQ persons surveyed were afraid of loss of sexual identity, inability to have their same sex partner recognized and lack of privacy (28). Many states do not provide legal protections for LGBTQ people, and the absence 
of these protections leave them with limited options in housing and care. The staff views highlighted in Di Napoli et. al.'s study (7) provides evidence that staff education and policies regarding the sexual rights of residents must be inclusive to all. Without policies and staff education that explicitly affirm sexual health and complete protections for LGBTQ individuals, these residents will continue to be at risk for discrimination and abuse at the hands of their caregivers.

\section{Ethical Considerations and Assessing for Consent}

Long-term residential care staff have an obligation to provide care that supports the well-being of each resident while also protecting them from harm. This tension is at the core of ethical dilemmas surrounding sexuality and dementia (25). In discussions of sexual health and dementia, it is important to note that older adults experiencing cognitive impairment are at risk for abuse. A 2006 study of 284 older adult victims of sexual abuse found that $60 \%$ of individuals in these cases were diagnosed with some form of dementia (29). Individuals with dementia can also be perpetrators of sexual abuse (25). A 2010 analysis found that sexual aggression against older adults is often reported in long-term care settings, and perpetrators of this abuse are often residents diagnosed with dementia (30). Wilkins (25) argues that given this information, long-term care facilities may create overly conservative approaches to sexuality among residents with dementia. But these restrictions of sexual expression would lead to further harm, infringing on resident autonomy, privacy, intimacy, pleasure, and creating circumstances that promote loneliness. This argument is valuable in educating residential care facilities with paternalistic practices about the negative impact sexual oppression has on the well-being of those living with dementia.

In the mild stage of dementia, individuals are able to communicate their needs clearly. Assessing for consent among these individuals could be a similar process to assessing consent among residents without cognitive impairment. When assessing for consent among those in the moderate and severe stages of the disease, a new way of thinking about consent is necessary as these stages are marked with difficulty in communication and decision making. While mental capacity and the ability to verbally communicate one's understanding of a situation are core components to decision making, they are not the sole characteristics in communicating consent for those living with dementia (22). Wilkins (25) explains that dementia does not equate to "global incapacity". While an individual could score low on a mental status test, such as the Mini-Mental State Examination (MMSE) or the SLU Mental Status Examination (SLUMS), they may still display task-specific competence $(22,25)$. While mental capacity examinations could define an individual as incompetent in decision making, their "preference for a special friend or lover may be quite evident". Wilkins (25) adds to the argument by noting that research has found the MMSE is not always a reliable predictor of mental capacity for those with dementia. These arguments provide evidence that there is no one way to measure capacity to consent. Because of the discrepancies of dementia symptoms between individuals, consent capacity should be assessed in a way that is tailored to the individual.

As dementia progresses, identity can also begin to shift. It is important to consider this when determining how to handle issues of consent and supporting the autonomy and sexuality of those experiencing dementia. Victor et al (27) define this transition as the "twilight of identity". Confusion and concern from family and health care providers can occur when an individual diagnosed with dementia begins to express themselves in a sexual way that is different from how they were pre-diagnosis $(25,27)$. Sexual advanced directives have been proposed as an intervention to assist in consent issues and changes in sexual behavior that can arise as dementia progresses (31). A sexual advanced directive could be completed at diagnosis or earlier, and the individual with dementia would have the ability to decide how they would like to be able to express their sexuality once in a long-term care setting. While Boni-Saenz (31) views sexual advanced directives as a way to protect the sexual rights of those with dementia, it is also possible that this tool could be used to suppress sexuality. There is no guarantee that the sexual advanced directive would be respected by a long-term care facility if they do not have explicit protections for resident sexuality written into their facility policy. The "twilight of identity" also creates an issue for sexual advanced directives. Because dementia may cause a change in one's personality and behaviors, creating a sexual advanced directive in a prediagnosis state could restrict one's sexual development and pleasure as the disease progresses.

Often when situations of sexual consent capacity arise in long-term care settings, facility staff and family members of the resident will meet to decide if the person with dementia should be permitted to continue their sexual expression. While committee-style assessments could be helpful in identifying capacity to consent by including multiple points of view when reviewing the sexual health and safety of an individual in the moderate to severe stages of dementia, this opens the door to potential biases and conflicting values that could harm the individual by suppressing their sexuality unnecessarily $(25,26)$. Family members often include those who have legal decisionmaking responsibilities for the residents with dementia, but healthcare staff cannot be positive that family members will act in the best interest of the person with dementia (25). Victor and Guidry-Grimes (27) argue:

"Unless the resident's sexual preferences endanger their well-being or the well-being of others based on an unbiased and careful assessment, and unless these behaviors cannot be easily managed by the care team, contacting family members or restraining the resident will carry heavy moral costs."

While family members or close friends have known the person with dementia longer than health care providers and 


\section{THE JOURNAL OF NUTRITION, HEALTH \& AGING}

facility staff, they may hold biases and judgments that could lead to restricting the resident's autonomy and access to pleasure.

Hindering the sexual expression of residents with dementia should only be done if the sexual activity causing harm to the individual or someone else. Those in the memory care field should take on a sex-positive and rights-based approach when considering capacity to consent for those with dementia. Evidence from the ethics research discussed previously indicates that promoting the sexual health and well-being of those with dementia is complex and should be considered on a case by case basis. Although individuals diagnosed with dementia experience different levels of mental capacity, this should not eliminate their right to explore sexuality within a long-term care setting. If the goal of long-term care facility staff is to support overall well-being and provide a person-centered care approach, then more facilities need to incorporate the sexual rights of residents into their policies, staff education, and practices.

The fundamental tenants of the BDSM (Bondage, Discipline/ Domination, Submission, and Sadism/Masochism) community include explicit consent, negotiation practices, and after care (32). These practices could be utilized to guide consent processes for sexual interactions that involve individuals with dementia. At this time, we could find no literature on guiding the consent process in older adults or those with dementia who practice BDSM.

\section{Facility Policies and Guidelines}

Residential facilities often have a resident bill of rights that must be given to residents and families upon admission. The 1987 Nursing Home Reform Act (NHRA) requires that facilities that receive Medicaid and Medicare payments must post a resident bill of rights in order to be in compliance with the policy (33). The Centers for Medicaid and Medicare services have expanded the 1987 NHRA bill of rights, but the amendments lack any mention of the sexual rights of residents (34). Without explicit protections under facility policies, residents in long-term care settings are at risk of being restricted from sexual expression. The Hebrew Home in Riverdale New York provides an example of a long-term care policy that provides guidelines, procedures, and staff and organizational responsibilities in protecting the sexual rights of their residents (22). In the document Policies and Procedures Concerning Sexual Expression at the Hebrew Home in Riverdale (35), the sexual rights of residents are explicitly stated. These rights include having a private space to sexually express yourself either alone or with a partner, access to sexually explicit materials, professional counseling pertaining to sexual health, and the right to engage in "Words, gestures, movements or activities (including reaching, pursuing, touching or reading) which appear motivated by the desire for sexual gratification.» This document, which was last revised in 2013, serves as a model of long-term care policy that advocates for residents' sexual rights.

In conjunction with written policies that include the sexual rights of residents, staff education on sexuality in older adulthood should utilize a sex-positive and sexual health life course perspectives and provide detailed guidelines on how to support resident sexual expression. A 2013 study measured the outcomes of an educational program delivered to long-term care nurses on sexuality and older adulthood (36). The program involved a 3-hour workshop that covered topics such as the impact of sexuality on well-being, barriers to sexual health in long-term care settings, the role of healthcare providers in the sexual health of older adults, and sexuality and dementia. Participants were given pre and post questionnaires to evaluate their knowledge and attitudes related to sexuality in older adulthood. The study's findings reported that the program had a significant positive impact on the permissiveness of staff attitudes towards sexuality in older adulthood, sexuality in residential care, and sexuality of those with dementia (36). This study provides evidence that staff education has the potential to improve attitudes towards sexuality among older adults and those living with dementia. Educating those who work directly with residents, who without training may curb residents' sexuality, is imperative in creating a sex-positive and safe environment for those with dementia who want to continue expressing themselves sexually.

Disclosures: Dr. Morley as having no conflicts to declare.

\section{References}

1. Lindau ST, Schumm LP, Laumann EO, et al. A study of sexuality and health among older adults in the United States. New England Journal of Medicine 2007;357:762774.

2. Huang AJ, Subak LL, Thom DH, et al. Sexual function and aging in racially and ethnically diverse women. JAGS 2009;57:1362-1368.

3. Smith L, Grabovac I, Yang L, et al. Participation in physical activity is associated with sexual activity in older English adults. Int J Environ Res Publ Health 2019;16:489-503.

4. Jannini EA, Nappi RE. Couplepause: A new paradigm in treating sexual dysfunction during menopause and andropause. Sex Med Rev 2018;6:384-395.

5. Benbow SW, Beeston D. Sexuality, aging, dementia. International Psychogeriatrics 2012;24:1026-1033.

6. Smith L, Yang L, Veronese N, et al. Sexual activity is associated with greater enjoyment of life in older adults. Sexual Medicine 2019;7:11-18.

7. Di Napoli EA, Breland GL, Allen RS. Staff knowledge and perceptions of sexuality and dementia of older adults in nursing homes. J Aging and Health 2013;25:10871105 .

8. Loeb DF, Lee RS, Binswanger IA, Ellison MC, Aagaard EM. Patient, Resident Physician, and Visit Factors Associated with Documentation of Sexual History in the Outpatient Setting. Journal of General Internal Medicine. 2011;26(8):887-893.

9. Gott M, Hinchliff S, Galena E. General practitioner attitudes to discussing sexua health issues with older people. Social Science \& Medicine. 2004;58(11):2093-2103

10. Bassil N, Morley JE. Late-life onset hypogonadism: A review. Clin Geriatr Med 2010;26:197-222.

11. Morley JE. The politics of testosterone. J Sex Med 2007;4:554-557.

12. Morley JE, Perry HM 3rd. Androgens and women at the menopause and beyond. J Gerontol A Biol Sci Med Sci 2003;58:M409-M416.

13. Morley JE. Impotence in older men. Hosp Pract (Off Ed) 1988;23:139-142

14. Boolell M, Allen MJ, Ballard SA, et al. Sildenafil: An orally active type 5 cyclic GMP-specific phosphodiesterase inhibitor for the treatment of penile erectile dysfunction. Int J Impot Res 1996;8:47-52.

15. Gong B, Ma M, Xie W, et al. Direct comparison of tadalafil with sildenafil for the treatment of erectile dysfunction: A systematic review and meta-analysis. Int Urol Nephrol 2017;49:1731-1740.

16. Wentzell E. How did erectile dysfunction become "Natural"? A review of the critical 


\section{SEXUALITY, AGING, AND DEMENTIA}

social scientific literature on medical treatment for male sexual dysfunction. J Sex Res 2017;54:486-506.

17. Irwin GM. Erectile dysfunction. Primary Care 2019;46:249-255.

18. Mobley DF, Khera M, Baum N. Recent advances in the treatment of erectile dysfunction. Postgrad Med J 2017;93:679-685.

19. Lindau ST, Dale W, Feldmeth G, et al. Sexuality and cognitive status: A U.S. nationally representative study of home-dwelling older adults. J Am Geriatr Soc 2018;66:1902-1910.

20. Holmes D, Reingold J, Teresi J. Sexual expression and dementia. Views of caregivers: A pilot study. International J Geriatr Psychiat 1997;12:695-701.

21. Davies HD, Sridhar SB, Newkirk LA, et al. Gender differences in sexual behaviors of AD patients and their relationship to spousal caregiver well-being. 2012;16:89-101.

22. Kuhn D. Intimacy, sexuality, and residents with dementia. Alzheimer's Care Q 2002;3:165-176.

23. Alagiakrishnan K, Lim D, Brahim A, et al. Sexually inappropriate behavior in demented elderly people. Postgraduate Med J 2005;81(957):463-466.

24. Canevelli M, Lucchini F, Garofalo C, et al. Inappropriate sexual behaviors among community-dwelling patients with dementia. The Am J Geriatr Psychiat 2017;25:365371 .

25. Wilkins JM. More than capacity: Alternatives for sexual decision making for individuals with dementia. The Gerontologist 2015;55:716-723.

26. Lester PE, Kohen I, Stefanacci RG, Feuerman M. Sex in nursing homes: A survey fo nursing home policies governing resident sexual activity. J Am Med Dir Assoc 2016;17:71-74
27. Victor E, Guidry-Grimes L. Relational autonomy in action: Rethinking dementia and sexuality in care facilities. Nursing Ethics 2019;26:1654-1664.

28. Mahieu L, Cavolo A, Gastmans C. How do community-dwelling LGBT people perceive sexuality in residential aged care? A systematic literature review. Aging Ment Health 2019;23:529-540.

29. Burgess A, Phillips S. Sexual abuse, trauma, and dementia in the elderly: A retrospective study of 284 cases. Victims and Offenders 2006;1:193-204.

30. Rosen T, Lachs MS, Pillemer K. Sexual aggression between residents in nursing homes: Literature synthesis of an underrecognized problem. J Am Geriatrics Soc 2010;58:1970-1979.

31. Boni-Saenz A. Sexual advance directives. Alabama Law Review 2016;68:48.

32. Pitagora, D. Consent vs. Coercion: BDSM Interactions Highlight a Fine but Immutable Line. The New School for Social Research 2013; 10:1: 27-36

33. Klauber M, Wright B. The 1987 Nursing Home Reform Act. 2001 https://www.aarp. org

34. Centers for Medicare and Medicaid Services. Your Rights and Protections as a Nursing Home Resident. n.d. https://downloads.cms.gov/

35. Dessel. R, Remiriz, M. Policies and Procedures Concerning Sexual Expression at the Hebrew Home in Riverdale. 1995. http://howieswebs.ipage.com/pdf/health/ sexualexpressionpolicy.pdf

36. Bauer M, McAuliffe L, Nay R, Chenco C. Sexuality in older adults: Effect of an education intervention on attitudes and beliefs of residential aged care staff. Educ Gerontol 2013;39:82-91. 